\title{
Injury-induced innate immune response in human skin mediated by transactivation of the epidermal growth factor receptor
}

\author{
Ole E. Sørensen,, ${ }^{1}$ Dharma R. Thapa, ${ }^{2}$ K. Markus Roupé,, ${ }^{1}$ Erika V. Valore, ${ }^{2}$ \\ Ulf Sjöbring, ${ }^{1}$ Alice A. Roberts, ${ }^{2}$ Artur Schmidtchen, ${ }^{3}$ and Tomas Ganz ${ }^{2}$

\begin{abstract}
${ }^{1}$ Section of Clinical and Experimental Infection Medicine, Department of Clinical Sciences, Lund University, Lund, Sweden. ${ }^{2}$ Host Defense Research Laboratory, Department of Medicine and Pathology, David Geffen School of Medicine at UCLA,
\end{abstract} \\ Los Angeles, California, USA. ${ }^{3}$ Department of Dermatology and Venereology, Lund University, Lund, Sweden.
}

\begin{abstract}
We found that sterile wounding of human skin induced epidermal expression of the antimicrobial (poly)peptides human $\beta$-defensin-3, neutrophil gelatinase-associated lipocalin, and secretory leukocyte protease inhibitor through activation of the epidermal growth factor receptor. After skin wounding, the receptor was activated by heparin-binding epidermal growth factor that was released by a metalloprotease-dependent mechanism. Activation of the epidermal growth factor receptor generated antimicrobial concentrations of human $\beta$-defensin-3 and increased the activity of organotypic epidermal cultures against Staphylococcus aureus. These data demonstrate that sterile wounding initiates an innate immune response that increases resistance to overt infection and microbial colonization.
\end{abstract}

\section{Introduction}

The skin and other epithelia not only serve as simple physical barriers against infection but can also mount an innate immune response by producing antimicrobial (poly)peptides (AMPs) to eliminate invading microbes (1). AMPs are widely distributed effector molecules of the innate immune response from insects to humans (2) and are active against a broad spectrum of Gram-positive and Gram-negative bacteria as well as some fungi and enveloped viruses, including HIV-1.

The important role of AMPs for the antimicrobial defense of skin and other epithelial sites is made evident by the increased susceptibility to epithelial infection of mice with disrupted AMP genes (3-5). Conversely, overexpression of AMPs leads to increased resistance against microbial infections at epithelial sites, including the skin (6-8). The role of AMPs for the host defense in human skin is also suggested by studies of 2 major human skin diseases characterized by defective skin barriers, atopic dermatitis and psoriasis. Patients with atopic dermatitis are much more prone to skin infections than patients with psoriasis. This difference has now been linked to the high expression of AMPs in psoriasis compared with atopic dermatitis (9-11).

Many studies have addressed how infection leads to the induction of the expression of AMPs in the skin $(12,13)$. However, despite the large number of potential infectious microbes encountered by humans, overt infection is a rare event. Thus, one of the functions of AMPs in the innate immune system may be not only to combat early or established infections but also to prevent infections from developing in the first place. To prevent infections, the

Nonstandard abbreviations used: AMP, antimicrobial (poly)peptide; AU-PAGE, acid urea-PAGE; hBD, human $\beta$-defensin; HB-EGF, heparin-binding EGF; IHC, immunohistochemistry; mBD-14, murine $\beta$-defensin-14; NGAL, neutrophil gelatinase-associated lipocalin; qRT-PCR, quantitative RT-PCR; SLPI, secretory leukocyte protease inhibitor; TAPI-1, tumor-necrosis factor $\alpha$ protease inhibitor-1; TBS, tris-buffered saline; TSB, trypticase soy broth.

Conflict of interest: The authors have declared that no conflict of interest exists. Citation for this article: J. Clin. Invest. 116:1878-1885 (2006). doi:10.1172/JCI28422. innate immune system must sense "high-risk situations" and activate the generation of AMPs. One such high-risk situation would be wounding, where the normal physical barrier of intact skin protecting against microbes is disrupted. Although wounding is commonly associated with simultaneous introduction of microbes, even a sterile wound provides an entry site for subsequent infection. Indeed, sterile wounding has been found to induce expression of AMPs in Drosophila through unknown mechanisms (14).

In this study, we examined the expression of AMPs in human skin after sterile wounding and found that AMPs were induced through transactivation of EGFR. Transactivation of EGFR is closely linked to reepithelization of skin wounds during the normal wound-healing process. Furthermore, we found that the antibacterial activity of epidermis was increased by activation of EGFR and that the concentrations of AMPs in epidermis of wounded skin exceeded those necessary for antimicrobial activity. Thus, wounding activates host defense mechanisms in the epidermis that can prevent harmful microbial colonization and infection.

\section{Results}

To establish whether sterile wounding induced the expression of AMPs in human skin, we developed a model of sterile-wounded human skin in culture. Healthy human skin fragments obtained as surgical residua were sliced into $1-\times 10-\mathrm{mm}$ slices and incubated in keratinocyte medium under sterile conditions. On days 0 , $1,2,3$, and 4, samples were processed for immunohistochemistry (IHC), RNA purification, or protein extraction. We examined the expression of the 3 human $\beta$-defensins (hBDs) present in skin, hBD-1 (15), hBD-2 (16), and hBD-3 (17). By Northern blotting, large amounts of hBD-3 mRNA were detected in the wounded skin at day 4 (Figure 1A), and by IHC, hBD-3 peptide was also found in the keratinocytes on day 4 (Figure 1B). The most intense staining for hBD-3 was around the wound edges of the skin slices. To further substantiate the induction of hBD-3 at the peptide level, extracts from skin from days 0 and 4 after wounding were analyzed by acid urea-PAGE (AU-PAGE), followed by blotting 
A

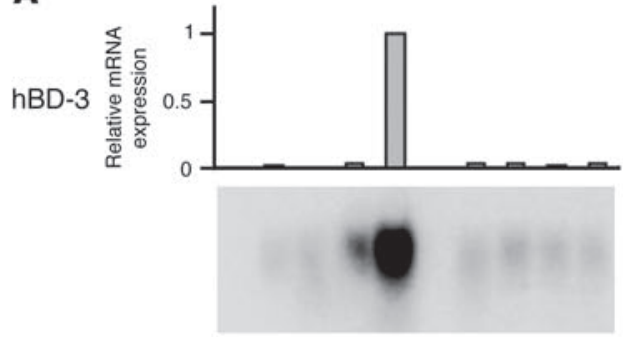

NGAL

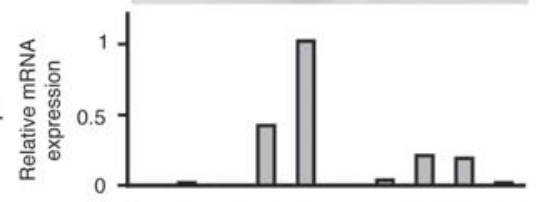

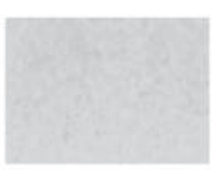

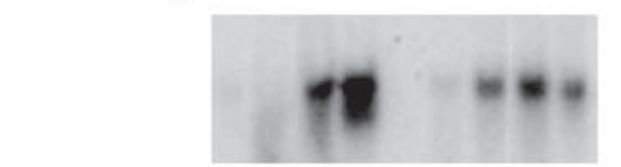

SLPI
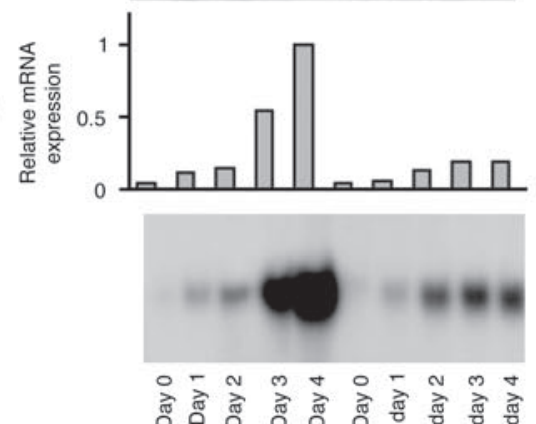

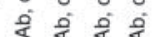
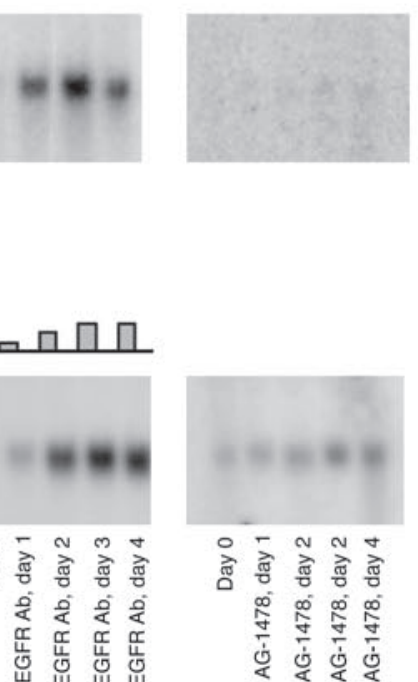

B
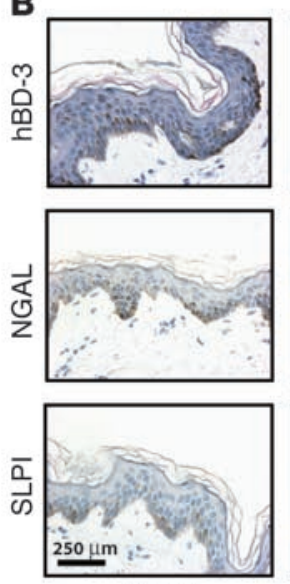

Day 0

C
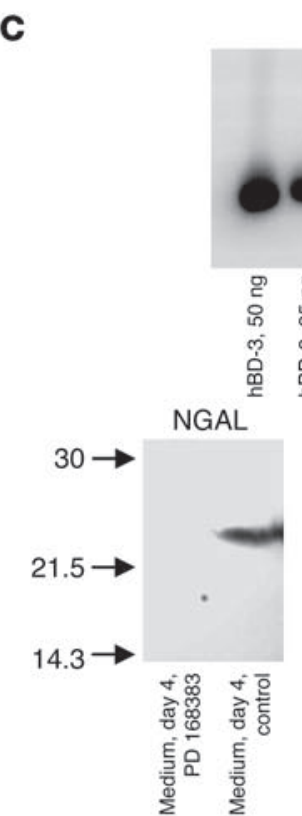

hBD-3
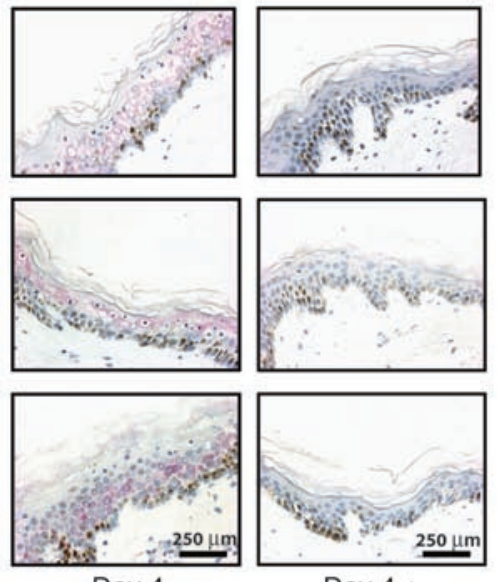

Day $4+$

AG-1478

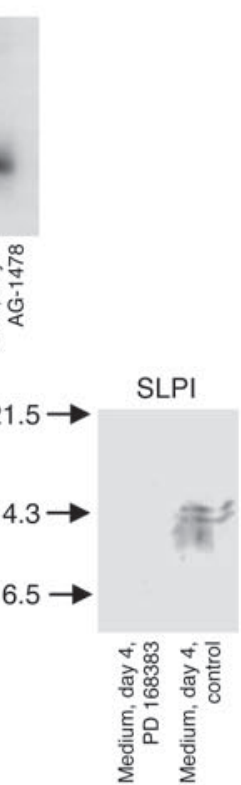

\section{Figure 1}

Expression of hBD-3, NGAL, and SLPI in wounded human skin. Human skin was sliced into 1- $\times 10-\mathrm{mm}$ slices and incubated for 0-4 days in culture. Each day, samples were processed for IHC or extracted for RNA or protein. (A) Northern blots of total RNA from whole skin. The blots were hybridized with probes for hBD-3, NGAL, SLPI, and G3PD. Graphs show the expression of hBD-3, NGAL, and SLPI normalized to the expression of G3PD mRNA. hBD-3, NGAL, and SLPI mRNA concentrations in the skin on day 4 were assigned the value 1. (B) Skin slices on days 0 and 4 with and without treatment with AG-1478 were stained for hBD-3, NGAL, and SLPI. Color was developed with fast red chromogen, and Harris hematoxylin was used for counterstaining. (C) The proteins extracted from both the wounded skin and the medium were analyzed by AU-PAGE and SDS-PAGE using synthetic hBD-3 as a standard and then blotted and probed with antibodies to hBD-3, NGAL, and SLPI. hBD-3 was only found in the skin. In contrast, NGAL and SLPI were only detected in the culture medium of the wounded skin. SLPI migrated as a double band around $14 \mathrm{kDa}$. This double band was not found in all samples tested and is probably due to proteolytic cleavage of SLPI during the preparation of this particular sample. AU-PAGE was used for detection of hBD-3 since this method is superior for examining small cationic peptides.

with anti-hBD-3 antibody. Only small amounts of hBD-3 were found in normal skin at day 0 , but the level was greatly increased by day 4 (Figure 1C). In contrast, we did not find induced expression hBD-1 and hBD-2 in the wounded human skin by Northern blots or IHC (data not shown).

To examine whether a simple breach of the epithelial lining of the skin was sufficient to induce the expression of hBD-3, we wounded keratinocyte organotypic epidermal cultures by sterile incision with a scalpel. After 4 days, there was intense staining for
hBD-3 peptide around the edges of the incision compared with the nonwounded cultures (Figure 2).

We also found that 2 other antimicrobial proteins present in human skin, neutrophil gelatinase-associated lipocalin (NGAL, also called siderocalin) (18) and secretory leukocyte protease inhibitor (SLPI) (19), were induced in our model along with hBD-3 (Figure 1, A-C). In accordance with previous findings, the basal expression of SLPI in the skin was low (19). SLPI was previously found to be induced in skin after wounding, through unknown mechanisms $(19,20)$. 


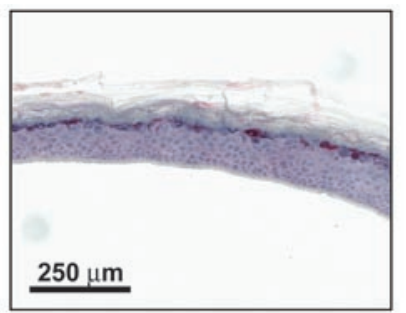

Epidermal culture

Nonwounded

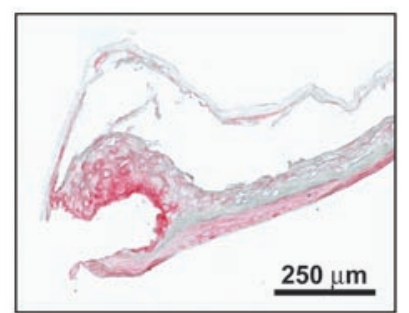

Epidermal culture Wounded

\section{Figure 2}

Expression of hBD-3 in wounded organotypic epidermal cultures. Organotypic keratinocyte epidermal cultures were wounded by sterile incision with a scalpel. Four days after wounding, the wounded and nonwounded samples were immunostained for hBD-3. Color was developed with fast red chromogen, and Harris hematoxylin was used for counterstaining.

To validate that our ex vivo wound model reflected wounding in vivo, we performed sterile wounding experiments in mice. We analyzed the expression of the murine orthologs of SLPI and NGAL (named 24p3 in mice) after sterile wounding of skin in mice and found that both these AMPs were induced 2 days after sterile wounding (Figure $3 \mathrm{~A}$ ). An ex vivo model of wounded mouse skin in culture showed a similar induction of $24 \mathrm{p} 3$ and SLPI (Figure 3B). Thus, the induction of AMPs in the ex vivo wound model reflected the induction after wounding in vivo. Not surprisingly, we found that induction of AMPs in mouse skin in vivo was lower than in the ex vivo model. This is likely due to the fact that in the ex vivo model, the skin is "wounded" around all the edges whereas in the in vivo, wounding only affects the smaller central part of the skin sample.

While the functional murine correlate of hBD-3 has not been identified, murine $\beta$-defensin-14 (mBD-14) has been suggested as the ortholog of hBD-3 due to conserved primary sequence. However, $\mathrm{mBD}-14$ was neither expressed in mouse skin nor induced by wounding, judged by quantitative RT-PCR (qRT-PCR) (data not shown).

To investigate whether the expression of hBD-3 peptide was induced after wounding in vivo, we analyzed human cutaneous wounds by IHC. Staining for hBD-3 was only found in the keratinocytes of the epidermis 4 days after the surgical wounding, with especially intense staining around the edges of the wound area (Figure 4).

In concert, the mouse experiments and the analysis of human cutaneous wounds confirmed that our ex vivo wound model reflected the in vivo situation.

We previously found that hBD-3, NGAL, and SLPI can be induced by activation of EGFR (21). To examine whether the increased expression of hBD-3 in wounded skin is dependent on activation of EGFR, the ex vivo-wounded human skin was incubated with AG-1478 or PD 168393, both specific inhibitors of EGFR signaling (Figure 1). AG-1478 completely abolished the induced expression and peptide production of hBD-3 (Figure 1A). Similar results were obtained with PD 168393 (data not shown). The expression of hBD-3 was also strongly inhibited by blocking antibodies against EGFR (Figure 1A), thus confirming that expression of hBD-3 in wounded skin was induced by activation of EGFR. Similarly, NGAL and SLPI were increased in the wounded skin in an EGFR-dependent manner (Figure 1A). The EGFR-dependent expression of hBD-3, SLPI, and NGAL in wounded skin was validated at the peptide/protein level by IHC and by Western blots of cultured skin and of the medium in which the skin was incubated (Figure 1, B and C). Increased levels of hBD-3 were found in extract from the skin. In contrast, increased levels of SLPI and NGAL were found in the medium from culture of the wounded skin. This probably reflects that SLPI and NGAL, in contrast to hBD-3, were secreted from the keratinocytes. Both IHC and Western blots showed that the induced expression of all 3 (poly)peptides on day 4 was abolished by the EGFR signaling inhibitors AG-1478 and PD 168393 (Figure 1, B and C).

We next analyzed the mRNA concentrations of woundinginduced AMPs by real-time qRT-PCR and found a generally large but highly variable induction of hBD-3 from day 0 to day 4 (Figure 5). We suspect that the variation was due to baseline expression of hBD-3, which is affected by preoperative exposure of the skin samples to (micro)trauma and microbial stimuli.

In approximately one-third of the donors, we observed much less pronounced induction of hBD-3 on Northern blot and only 10- to 15 -fold induction by qRT-PCR. In these "nonresponders," the hBD-3 mRNA concentration at day 4 was always much lower than the concentration of G3PD mRNA. In contrast, in the "responders," hBD-3 mRNA concentrations were higher than those of G3PD mRNA at day 4. Due to the restrictions imposed by the Institutional Review Boards, we were not able to investigate the reasons for the diminished response in some donors. Possibilities include the age of the patients, medication, the handling of samples, and poor wound healing.

To determine the molecular events that led to the activation of EGFR and production of AMPs in wounded human skin, we subsequently focused on hBD-3. We previously found that several EGFR ligands were capable of inducing hBD-3 in keratinocytes (13). Accordingly, we examined whether EGFR or any of its ligands were induced prior to hBD-3 after wounding. Using real-time qRTPCR, we found no increase in EGFR mRNA or in mRNA encoding
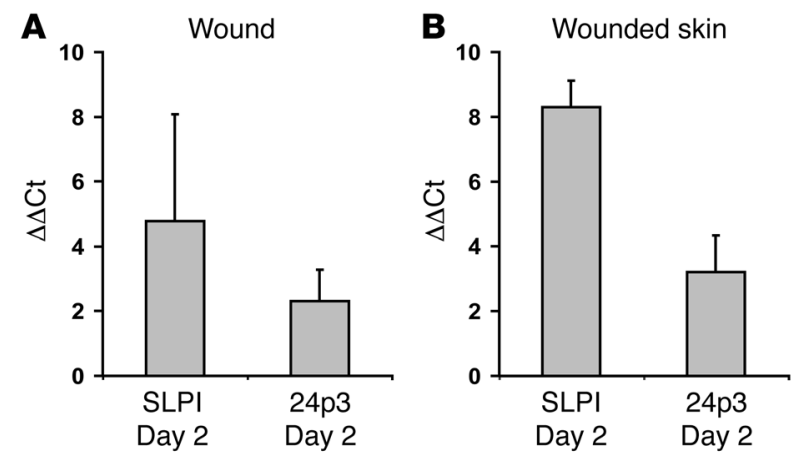

\section{Figure 3}

Wound experiments in mice. (A) Mice $(n=5)$ were wounded by sterile incisions. Two days later, the mice were sacrificed and the RNA was extracted from the wounded skin surrounding the cut. The mRNA expression of the murine orthologs of NGAL (24p3) and SLPI was analyzed by real-time qRT-PCR and shown as the difference in threshold cycles $(\mathrm{Ct})$ between the gene of interest and $\beta$-actin as housekeeping gene control. The expression of $24 \mathrm{p} 3$ and SLPI in nonwounded skin was set to 0 . Thus, the bars at day 2 depict the induction of SLPI and $24 \mathrm{p} 3$ as shown in changes of threshold cycles $(\Delta \Delta \mathrm{Ct})$. (B) Mouse skin was sliced into $1 \times 10-\mathrm{mm}$ slices and incubated for 2 days in culture $(n=4)$. RNA was extracted from the skin. The expression of $24 \mathrm{p} 3$ and SLPI was analyzed by real-time qRT-PCR. The mRNA expression is shown as the difference in threshold cycles between the gene of interest and $\beta$-actin as housekeeping gene control $(\Delta \delta \mathrm{Ct})$. The expression of $24 \mathrm{p} 3$ and SLPI in nonwounded skin was set to 0 so that the bars at day 2 depict the induction of SLPI and 24p3. 
Normal skin, day 0
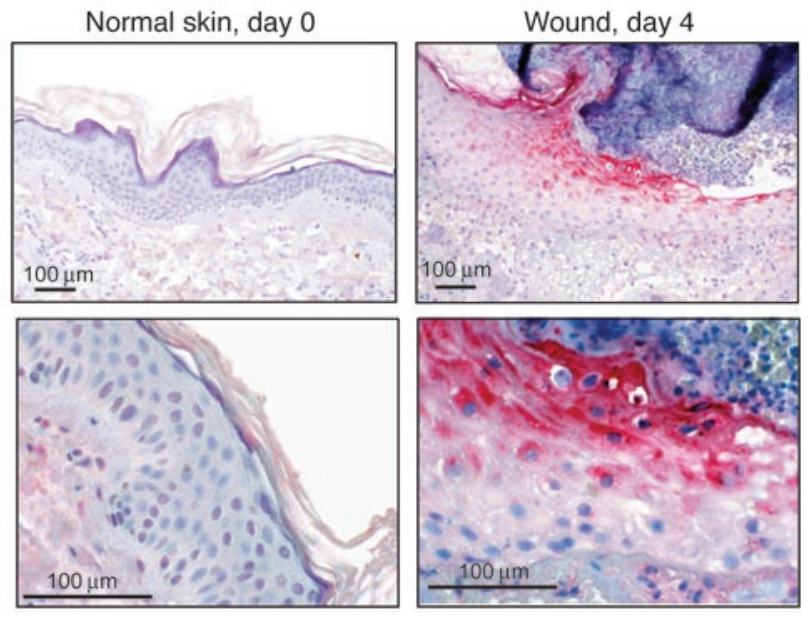

Figure 4

hBD-3 expression in a cutaneous wound. Samples of normal human skin and skin from a 4-day-old wound were immunostained for hBD-3. Color was developed with fast red chromogen, and Harris hematoxylin was used for counterstaining.

its ligands in the wounded skin (Figure 6). Therefore, EGFR-dependent induction of hBD-3 was not a result of induced expression of EGFR mRNA or the mRNA of any of its known ligands in the wounded skin. However, in all samples analyzed, heparin-binding EGF (HB-EGF) was consistently the EGFR ligand with the highest expression in the skin (see Table 1).

Membrane-bound EGFR ligands can be released by activated metalloproteases that mediate ectodomain shedding from epithelial cells. The released growth factors are then able to bind and activate the EGFR (22), a process referred to as transactivation of EGFR. Members

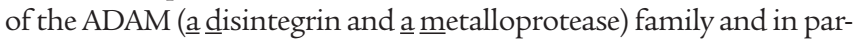
ticular ADAM-17, also known as tumor-necrosis factor $\alpha$ converting enzyme (TACE), have been implicated in the transactivation process. To test whether induction of hBD-3 was caused by transactivation of EGFR, the ex vivo-wounded skin was incubated with a TACE inhibitor, tumor-necrosis factor $\alpha$ protease inhibitor-1 (TAPI-1). TAPI-1 inhibited the expression of hBD-3 (Figure 7A). In contrast, inhibitors of serine proteases (aprotinin) or cysteine proteases (E-64) did not affect the expression of hBD-3 in wounded skin (data not shown).

To identify the EGFR ligand(s) responsible for the hBD-3 expression, wounded skin was incubated with blocking antibodies against the EGFR ligands TGF- $\alpha$ and HB-EGF(Figure 7B). These 2 growth factors are the most highly expressed EGFR ligands in the skin (Table 1), and they are the most potent inducers of hBD-3 (13). Blocking antibodies against HB-EGF but not to TGF- $\alpha$ partially inhibited the expression of hBD-3 mRNA. To verify the role of HB-EGF in the induction of hBD-3, wounded skin was incubated with CRM197, a nontoxic analogue of diphtheria toxin that specifically binds to and inhibits the release of membrane-bound HB-EGF (23) but does not inhibit the effect of soluble HB-EGF or any of the other EGFR ligands. The addition of CRM197 inhibited the induction of hBD-3 mRNA (Figure 7C), and both TAPI-1 and CRM197 also inhibited hBD-3 peptide expression as detected by IHC (Figure 8 ). Thus, the increase of hBD-3 concentration in wounded skin is mediated by HB-EGF in wounded skin by transactivation of EGFR.

After wounding, approximately $50 \mathrm{ng}$ of hBD-3 was detected in the extract from $0.15-\mathrm{cm}^{2}$ skin on day 4 (Figure 1C). Assuming that the thickness of the epidermis is around $0.25 \mathrm{~mm}$ (Figure 8), this gives a concentration of hBD-3 of approximately $13 \mu \mathrm{g} / \mathrm{ml}$. Since the most intense staining for hBD-3 was found around the wounded edges and in the upper layers of epidermis, the local concentrations of hBD-3 in these areas are probably much higher than the concentration in the whole epidermis.

As the estimated concentration of hBD-3 found in whole epidermis was above the concentration of hBD-3 required for killing of the important skin pathogen Streptococcus pyogenes (13), we investigated whether the activation of EGFR could increase the overall antibacterial activity of epidermis. Organotypic epidermal cultures were stimulated with TGF- $\alpha$ and then extracted for analysis in antibacterial assays. Epidermis contains prominent antibacterial activity against Escherichia coli (24). To test the efficiency of the extraction of AMPs from epidermis, we examined the activity of the epidermal extracts against E. coli and found, as expected, prominent activity against E. coli in the extracts from both nonstimulated and TGF- $\alpha$-stimulated epidermal cultures. In contrast, and in accordance with previous findings (25), extracts from the nonstimulated epidermal cultures did not show significant antibacterial activity against Staphylococcus aureus compared with the buffer control (Figure 9). However, extracts of epidermal cultures stimulated with TGF- $\alpha$ had significantly increased antibacterial activity against $S$. aureus (Figure 9) compared with extracts from nonstimulated epidermal cultures or the buffer controls. Thus, the activation of EGFR with subsequent induction of AMPs following sterile wounding stimulates the antibacterial properties of the epidermis against a skin pathogen.

\section{Discussion}

We hypothesized that expression of AMPs may be induced in the skin after sterile wounding. Indeed, we found that sterile wounding induced the expression of 3 AMPs in human skin, hBD-3, NGAL, and SLPI. We previously found that the stimulation of human skin with

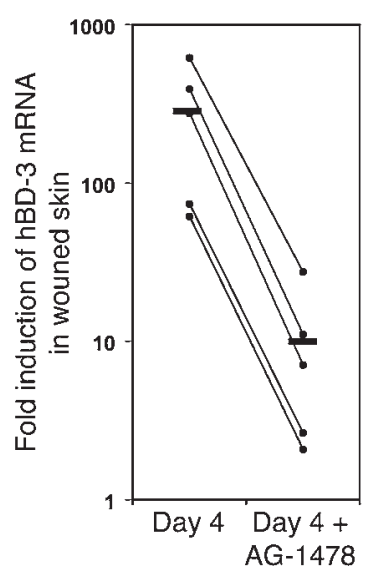

\section{Figure 5}

Fold induction of hBD-3 mRNA in ex vivo-wounded skin determined by qRT-PCR. The relative expression of hBD-3 mRNA was analyzed by realtime qRT-PCR on skin samples from 5 different donors. The expression of hBD-3 was normalized to the housekeeping gene G3PD. The treatment with AG-1478 did not influence the G3DP levels. The expression of hBD-3 at day 0 was set to 1 . Horizontal lines denote the average induction of the 5 donors. The donor with a 27-fold increase even after treatment with AG-1478 was the same donor who had a 631-fold induction of hBD-3. Thus, even in this donor, AG-1478 dramatically inhibited hBD-3 expression. The donors included in this figure were all responders. 


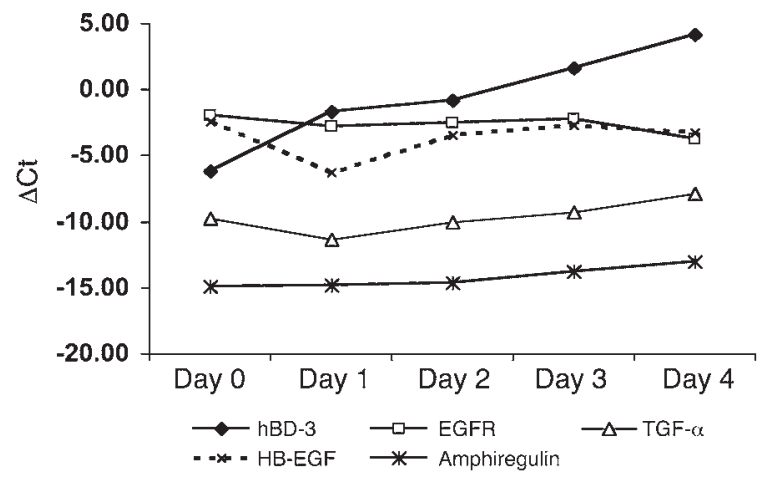

Figure 6

The expression of EGFR and EGFR ligands in wounded skin. The expression of mRNA of EGFR, TGF- $\alpha$, HB-EGF, amphiregulin, and hBD-3 was analyzed by real-time $\mathrm{qRT}$-PCR. The MRNA expression is shown as the difference in threshold cycles between the gene of interest and G3PD. The expression of EGFR and its ligands was stable over time.

microbe-derived molecules leads to induced expression of hBD-3 as well as 2 other $\beta$-defensins, hBD-1 and hBD-2 (13). The induction of AMPs after wounding was not due to inadvertent stimulation of the skin with microbes/microbe-derived molecules because we did not observe the induction of hBD-2 that is characteristic of microbial or cytokine stimulation. Thus, the increase of AMPs in wounded skin was selective and due to the wounding itself.

Transactivation of EGFR is an important regulator of reepithelization in wound healing $(26,27)$. HB-EGF was found to be released in wounded skin (28) and responsible for activation of EGFR in the skin (26). Inhibition of the transactivation process led to retarded reepithelization in vivo $(26,27)$ consistent with the key role of EGFR in epithelization and in wound healing (29). A simple breach of a monolayer of keratinocytes is sufficient for the initiation of this transactivation process (26). Similarly, we found that simple physi- cal disruption of the epithelial lining in organotypic epidermal keratinocyte cultures was sufficient to increase hBD-3. Thus, wounding or damage to epithelia leads to transactivation of EGFR and coordinated expression of AMPs during reepithelization of wounds.

To test whether activation of EGFR increased the antibacterial activity of the epidermis against potential skin pathogens, we stimulated activated EGFR in the defined setting of organotypic epidermal cultures of human keratinocytes. Stimulation of EGFR in the epidermal cultures resulted in antibacterial activity against the skin pathogen $S$. aureus, a microbe known to cause serious skin infections (30). In contrast, we found significant activity against $E$. coli even in nonstimulated epidermal cultures. This is not surprising since normal skin is very resistant to E. coli due to production of psoriasin, an antimicrobial protein with potent and selective activity against E. coli (24).

In our wound model, significant expression of AMPs was first observed 3-4 days after wounding. The first days after wounding are characterized by the influx of neutrophils, and these may be responsible for the initial clearance of microbes from the wound. However, the continued presence of neutrophils with their cytotoxic and proteolytic arsenal may not be conducive to wound healing, and the neutrophils disappear from the wound usually at 3-5 days after wounding (31). The increased expression of AMPs coincides with the disappearance of neutrophils and leads us to propose that epithelial AMPs are important for the antibacterial defense in the wound after the disappearance of the neutrophils and before the complete reestablishment of the physical barrier. We previously found that differentiation is an important determinant for expression of AMPs in keratinocytes (21, 32). In monolayer cultures of keratinocytes, we first found expression of AMPs in postconfluent cells (21). It is possible that the keratinocytes do not start to express AMPs until they have partially restored the epithelium in the wound and have begun to differentiate.

Interestingly, stimulated neutrophils diapedesed into skin windows release LL-37 (33), and this peptide has been shown to cause transactivation of EGFR (34). Thus, the neutrophils in the wounds may stimulate the subsequent expression of AMPs in the epidermis.
A

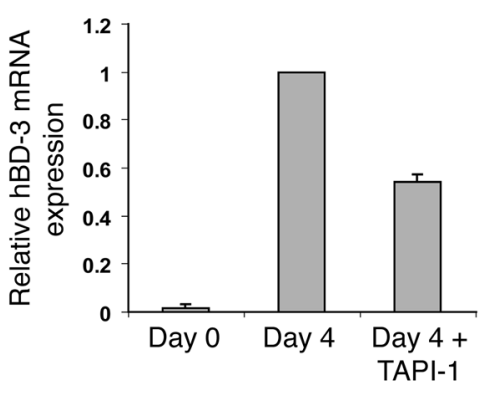

B

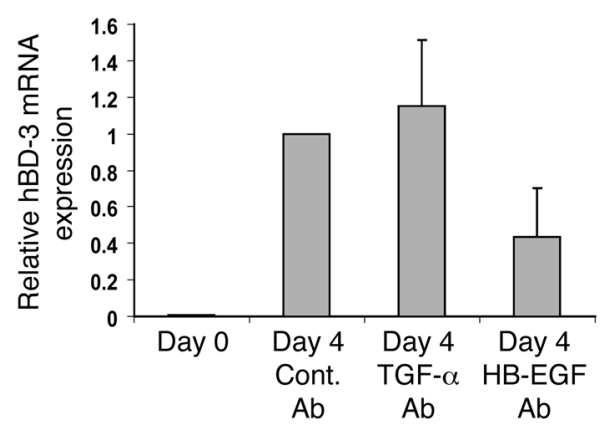

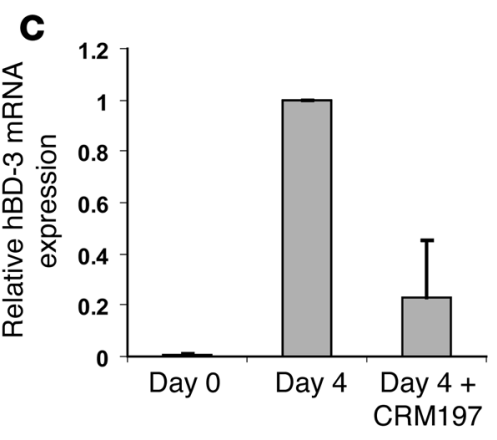

Figure 7

Identification of EGFR ligand responsible for hBD-3 induction in wounded skin. (A) Skin slices were incubated with the metalloprotease inhibitor TAPI-1 or with the DMSO vehicle used to dissolve the TAPI-1. Expression of hBD-3 was analyzed by real-time qRT-PCR on days 0 and 4 . The expression of hBD-3 at day 4 was set to 1 . TAPI- 1 inhibited the expression of hBD-3 compared with the DMSO vehicle $(n=3, P<0.002)$. Mean and standard deviation are shown. (B) Skin slices $(n=4)$ were incubated with control antibodies or antibodies against TGF- $\alpha$ and HB-EGF. The expression of RNA was analyzed by real-time qRT-PCR and normalized to G3PD as housekeeping mRNA control. The expression of hBD-3 on day 4 in the presence of control antibodies was set to 1. Due to limited access to whole skin, not all of the inhibition experiments were carried out each time. HB-EGF antibodies significantly inhibited the expression of hBD-3 compared with control antibodies $(P<0.003)$. Mean and standard deviation are shown. (C) Skin slices were incubated with an analog of diphtheria toxin, CRM197 $(n=3)$. CRM197 associates with membranebound HB-EGF and prevents its proteolytic liberation. The expression of mRNA was analyzed by real-time qRT-PCR and normalized to the G3PD as housekeeping mRNA control. The expression of hBD-3 on day 4 was set to 1. CRM197 significantly inhibited the expression of hBD-3 compared with control antibodies $(P<0.03)$. Mean and standard deviation are shown. 
Table 1

Expression of EGFR and EGFR ligands in wounded skin

\section{Expression in threshold cycles correlated to G3PD}

EGFR

TGF- $\alpha$

HB-EGF

Amphiregulin
Day 0

$$
\begin{gathered}
-2.3(-1.3 \text { to }-3.6) \\
-10.1(-9.9 \text { to }-10.3) \\
-2.5(-1.1 \text { to }-3.4) \\
-10.1(-5.4 \text { to }-12.5)
\end{gathered}
$$

Day 4

$$
\begin{array}{cc}
-4.9(-4.4 \text { to }-5.4) & -3.6(-2.6 \text { to }-4.6) \\
-8.7(-8.4 \text { to }-9.2) & -9.1(-7.8 \text { to }-10.3) \\
-2.3(-0.6 \text { to }-3.2) & -2.6(-2.2 \text { to }-3.5) \\
-10.5(-8.9 \text { to }-11.4) & -12.1(-10.8 \text { to }-13.0)
\end{array}
$$

Day 4 + AG-1478
The induction of EGFR and EGFR ligand mRNA in wounded skin was analyzed by real-time qRT-PCR with RNA from 3 different donors (the results from the donor mentioned in the Figure 5 legend are not included here). The expression is depicted as threshold cycles referenced to the threshold cycles of G3PD. Ranges of the threshold cycles are shown in parentheses.

medium from Cambrex supplemented with transferrin, hEGF $(0.15 \mathrm{ng} / \mathrm{ml})$, $0.5 \mathrm{mg} / \mathrm{ml}$ hydrocortisone, gentamicin, amphotericin B, and epinephrine but without insulin. We previously found that this medium does not induce the expression of AMP in keratinocytes (21). In the inhibition experiment, the skin slices were incubated with blocking antibodies at a final concentration of $15 \mu \mathrm{g} / \mathrm{ml}, 50 \mu \mathrm{M}$ TAPI-1 (Peptides International), $10 \mu \mathrm{g} / \mathrm{ml}$ CRM197 (Sigma-Aldrich), 0.2 trypsin inhibitory units of aprotinin (Sigma-Aldrich), and $5 \mu \mathrm{g} / \mathrm{ml} \mathrm{E-64}$ (Sigma-Aldrich).

Several studies have demonstrated that overexpression of AMPs in mice protects the animals against subsequent infection in the skin and other epithelial sites (6-8). Skin wounding represents a vulnerable state for subsequent infections where "preventive" expression of AMPs could be beneficial. Such preventive generation of AMPs is reminiscent of the sterile wounding response in Drosophila that includes the induction of several antimicrobial peptides (14). In frog skin, AMPs play a major role in preventing wound infection after nonsterile surgery (35), and other danger signals, such as electric stimuli or norepinephrine, result in the release large amounts of AMPs from serous glands in the skin (36). In this setting, even released neuropeptides may have a direct role as antimicrobials (37). In humans, circulating neutrophils with abundant amounts of AMPs are rapidly recruited to epithelial sites even in sterile inflammation and may provide early antimicrobial protection. Following sexual intercourse - another risk situation for microbial infection - AMPs are generated in the vagina by a microbe-independent mechanism from microbicidal precursor proteins present in seminal plasma (38). Thus, activation of antimicrobial mechanisms in situations associated with a high risk of infection may be a common feature of the innate immune response.

In conclusion, we found that transactivation of EGFR in wounded human skin leads to expression of AMPs and that activation of EGFR results in increased antibacterial activity of the epidermis. These data provide evidence for the concept that certain high-risk situations for infections alert the innate immune system in the skin even in the absence of microbes and induce alterations in the epidermis that prevent harm from microbial colonization and infection.

\section{Methods}

Reagents. The anti-hBD-1 and anti-hBD-2 antibodies were previously described $(32,39)$. Anti-hBD-3 antibodies were purchased from Orbigen or generated by immunization of rabbits with synthetic hBD-3 as previously described (13). Commercial antibodies were used for the IHC in Figures 1 and 2. Custom-made anti-hBD-3 antibodies were used in all other experiments. Synthetic hBD-3 was purchased from PeproTech. Alkaline phosphatase-conjugated goat anti-rabbit antibody was from Pierce Biotechnology. SLPI antibodies, control antibodies, and neutralizing antibodies against TGF- $\alpha$ and HB-EGF were purchased from R\&D Systems. Neutralizing antibodies against EGFR were obtained from EMD. The anti-NGAL antibodies were described previously (40). PD 168383 was purchased from EMD and AG-1478 from Sigma-Aldrich.

Skin specimens. Skin specimens were obtained as excess healthy tissue from skin surgery, under protocols approved by the Institutional Review Board at UCLA and the Ethics Committee at Lund University. The surgical specimens were cut into slices of $1 \times 10 \mathrm{~mm}$ and grown in serum-free keratinocyte
Human skin wounds. Samples from human skin wounds were obtained under protocols approved by the Ethics Committee at Lund University. A skin wound was induced by a punch biopsy on the upper arm of healthy male volunteers after informed consent. After 4 days, new punch biopsies were taken from the edges of the initial biopsy.

Extraction of AMPs from skin and medium. Skin slices were homogenized in $1 \mathrm{M} \mathrm{HCl}$ and incubated for 24 hours at $4^{\circ} \mathrm{C}$ under rotation, followed by centrifugation at $10,000 \mathrm{~g}$. The pellets were incubated 2 additional times with $5 \%$ acetic acid, followed by centrifugation at $10,000 \mathrm{~g}$. Supernatants were collected, lyophilized, and resuspended in $1 \mathrm{ml}$ of distilled $\mathrm{H}_{2} \mathrm{O}$. The resuspended supernatants were pooled and diluted to a total volume of $20 \mathrm{ml}$ in distilled $\mathrm{H}_{2} 0$. The $\mathrm{pH}$ was adjusted to 7 , and the sample was incubated at room temperature with MacroPrep CM Support beads (Bio-Rad) equilibrated in $25 \mathrm{mM}$ ammonium acetate ( $\mathrm{pH}$ 6.8) for 3-4 hours. The beads were subsequently washed, and the bound material was eluted with $5 \%$ acetic acid. The eluate was lyophilized and resuspended in $0.01 \%$ acetic acid and desalted and con-

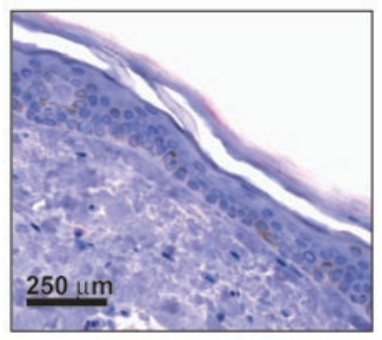

Day 0

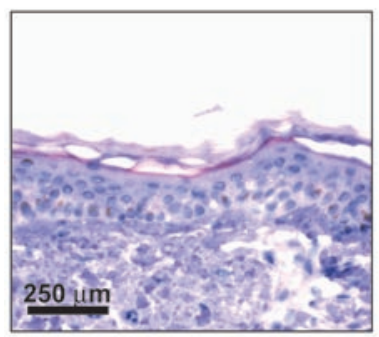

Day $4+$ CRM197

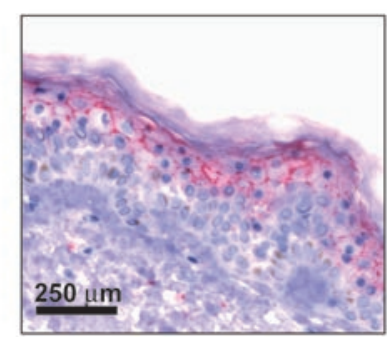

Day 4

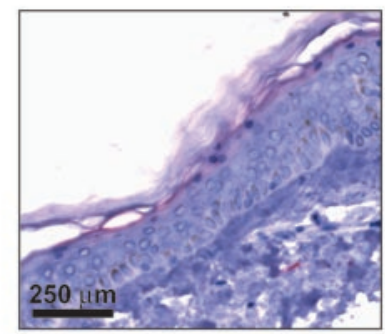

Day $4+$ TAPI-1

\section{Figure 8}

The effect of inhibitors on the expression of immunoreactive hBD-3 peptide in wounded skin. Skin slices on days 0 and 4 with and without treatment with TAPI-1, CRM197, and the vehicle for TAPI-1 (DMSO) were stained for hBD-3. Color was developed with fast red chromogen, CRM197 were found to inhibit the expression of hBD-3. and Harris hematoxylin was used for counterstaining. Both TAPI-1 and 

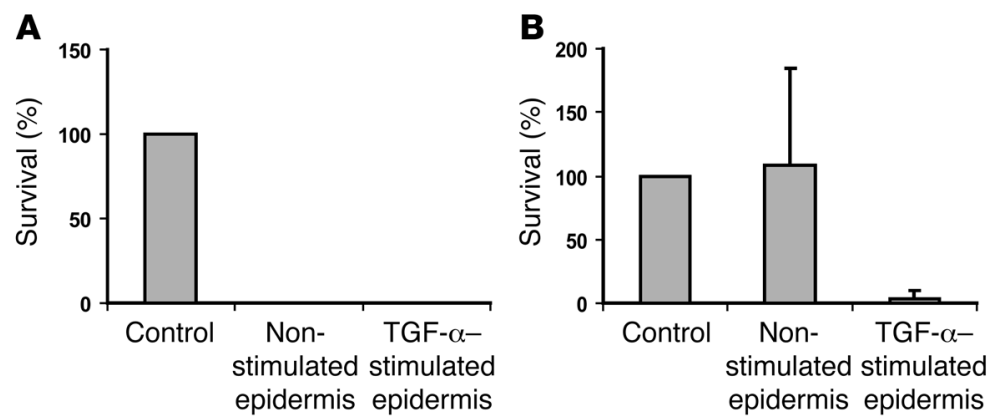

Figure 9

Antibacterial activity of extract from organotypic epidermal cultures. CFU assays using $E$. coli $(\mathbf{A})$ and $S$. aureus $(\mathbf{B})$ as targets were performed with extract from epidermal cultures and are displayed here as bacterial survival compared with control samples incubated with buffer alone. As expected, there was no difference in the killing of $E$. coli with nonstimulated epidermal cultures and TGF- $\alpha$-stimulated epidermal cultures $(P=0.4, n=3)$. In contrast, there was a significant difference in the killing of $S$. aureus between nonstimulated epidermal cultures and TGF- $\alpha$-stimulated epidermal cultures $(P<0.041, n=5)$. Nonstimulated epidermal cultures did not cause significant killing of $S$. aureus compared with buffer alone $(P=0.77, n=5)$. Mean and standard deviation are shown.

centrated using Microcon filter (Millipore) with molecular cutoff at $3 \mathrm{kDa}$. The retentate was lyophilized and resuspended in $50 \mu \mathrm{l} 0.01 \%$ acetic acid.

AU-PAGE, SDS-PAGE, and immunoblotting were performed according to the manufacturer's instructions (Hoefer). After transfer of proteins from the polyacrylamide gels, the PVDF membrane was fixed for 30 minutes in tris-buffered saline (TBS) with $0.05 \%$ glutaraldehyde (Sigma-Aldrich) and blocked with Superblock Blocking Buffer (Pierce Biotechnology). For visualization of the poly(peptides), the PVDF membranes were incubated overnight with primary Abs. The following day, the membranes were incubated for 2 hours with HRP-conjugated secondary Abs (Pierce Biotechnology) and visualized by Immun-Star HRP luminal/enhancer and Immun-Star peroxide buffer (Bio-Rad). The PVDF membrane was stripped for 20 minutes in $0.2 \mathrm{M}$ Glycine ( $\mathrm{pH} 2.5$ ) and $1 \%$ SDS, washed twice with TBS with $0.05 \%$ Tween 20 , and finally blocked before incubating overnight with a different antibody.

Stimulation and wounding of organotypic epidermal cultures. Primary epidermal cultures EPI-200-3S (MatTek Corp.) containing human epidermal keratinocytes were grown on collagen-coated Millicell CM Membranes (Millipore). The cultures were placed in 12-well plates with media (which contained no bovine pituitary extract) supplied by the manufacturer. On day 4 , the epidermal cultures were lifted to the air-liquid interface and then cultured in air-liquid interface for another 4 days according to the manufacturer's instructions. On day 2 after airlifting the cultures, the medium was changed to medium without insulin or EGF and without antibiotics. On day 4 after airlifting, the cultures were stimulated with TGF- $\alpha(50 \mathrm{ng} / \mathrm{ml})$. Cells were harvested after 48 hours of stimulation.

The cultures were homogenized in $1 \mathrm{M} \mathrm{HCl}$ and sonicated on ice 3 times for 10 seconds each time. The samples were incubated for 24 hours at $4{ }^{\circ} \mathrm{C}$ under rotation, followed by centrifugation at $10,000 \mathrm{~g}$. The supernatants were collected and lyophilized and resuspended in $400 \mu \mathrm{l}$ of distilled $\mathrm{H}_{2} \mathrm{O}$. The solution was desalted and concentrated using Microcon filter (Millipore) with a molecular cutoff at $3 \mathrm{kDa}$. The eluate was finally resuspended in $50 \mu \mathrm{l}$ of $0.01 \%$ acetic acid. This material was subsequently used for antibacterial assays. For the in vitro wounding experiments, EPI-200 cultures were used. The cultures were wounded by a sterile scalpel. Samples were processed for IHC 3 and 4 days after wounding.

RNA isolation. Total RNA was isolated with TRIzOL (Invitrogen) according to the recommendations of the manufacturer. The RNA was double purified with TRIzOL, then precipitated with ethanol and resuspended in $0.1 \mathrm{mM}$ EDTA. The concentration was determined by spectrophotometric measurement and the integrity of the RNA assessed by running a sample on an agarose gel.

Northern blotting. For Northern blotting, $5 \mu \mathrm{g}$ of RNA was analyzed by size on a $1 \%$ agarose gel with $6 \%$ formaldehyde dissolved in $1 \times$ MOPS $(20 \mathrm{mM}$ Mops, $5 \mathrm{mM}$ sodium acetate, and 1 mM EDTA, pH 7.0). The RNA was transferred to a Hybond-N membrane (Amersham Biosciences) by capillary blotting and fixed by UV irradiation. The filters were prehybridized for a minimum of 30 minutes at $42^{\circ} \mathrm{C}$ in $10 \mathrm{ml}$ ULTRAhyb (Ambion) and hybridized overnight at $42^{\circ} \mathrm{C}$ after addition of another $5 \mathrm{ml}$ ULTRAhyb containing the ${ }^{32} \mathrm{P}$-labeled probe. The membranes were washed twice for 5 minutes each time at $42^{\circ} \mathrm{C}$ in $2 \times \mathrm{SSC}(1 \times \mathrm{SSC}=150 \mathrm{mM} \mathrm{NaCl}$ and $15 \mathrm{mM}$ sodium citrate, $\mathrm{pH} 7.0), 0.1 \%$ SDS followed by 2 periods of 15 minutes each in $2 \times$ SSC, $0.1 \%$ SDS, 1 period of 15 minutes in $0.2 \times$ SSC, $0.1 \%$ SDS, and 1 period of 15 minutes in $0.1 \times$ SSC, $0.1 \%$ SDS at $42^{\circ} \mathrm{C}$. The blot was developed and then quantified by a phosphoimager (Bio-Rad). The sizes of the mRNAs were determined by reference to $18 \mathrm{~S}$ and $28 \mathrm{~S}$ ribosomal RNA, which were visualized by staining of membranes with methylene blue. The membranes were stripped by boiling in $0.1 \%$ SDS before rehybridization. The cDNA probes for hBD-3, NGAL, and SLPI were described previously (21), and the probe for G3PD was from Stratagene.

IHC. The skin slices were fixed in $10 \%$ formalin, dehydrated, and embedded in paraffin. Sections of $5-\mu \mathrm{m}$ thickness were placed on polylysine-coated glass slides, deparaffinized in xylene, and rehydrated in graded alcohols. The slides were then trypsinated for 15 minutes in $0.05 \mathrm{M}$ Tris ( $\mathrm{pH} 7.4$ ) with $0.5 \mathrm{mg} / \mathrm{ml}$ trypsin and $0.5 \mathrm{mg} / \mathrm{ml} \mathrm{CaCl}_{2}$ or treated with Dako antigen-retrieval solution for 40 minutes at $97^{\circ} \mathrm{C}$. The slides were incubated in a 1:1000 dilution of rabbit polyclonal antibodies against NGAL and SLPI and a 1:666 dilution of rabbit polyclonal antibodies against hBD-3. The antibodies were diluted in TBS with $1 \%$ BSA, $5 \%$ goat serum, $0.05 \%$ Tween 20 (Sigma-Aldrich), and $0.01 \%$ thimerosal, and the slides were incubated for 24 hours at room temperature. After 3 washes of 20 minutes each in TBS with $0.05 \%$ Tween 20, the slides were incubated with alkaline phosphatase-conjugated goat anti-rabbit IgG (Pierce Biotechnology) diluted 1:1000 in the same buffer as the first antibody and incubated for another 24 hours followed by 3 washes of 20 minutes each. Color was developed with Fast Red chromogen (Sigma-Aldrich) in Tris buffer, and the slides were counterstained with Harris Hematoxylin (EM Science).

Real-time PCR. cDNA was synthesized from 200 ng purified RNA using iScript cDNA synthesis kit (Bio-Rad) according to the manufacturers' instructions. hBD-1, hBD-2, and hBD-3 together with G3PD expression was analyzed using iQ SYBR Green Supermix (Bio-Rad). The primers were as follows: hBD-3: 5'CTTCTGTTTGCTTTGCTCTTCC- ${ }^{\prime}$ and $5^{\prime}$-CACTTGCCGATCTGTTCCTC3'; human G3PD: 5'-TGGTATCGTGGAAGGACTC-3' and 5'-AGTAGAGGCAGGGATGATG-3'; TGF- $\alpha$ : 5'-CTGGCTGTCCTTATCATCAC-3' and 5'-AGCGGTTCTTCCCTTCAG-3'; HB-EGF: 5'-TGCCAAGTCTCAGAAGAGG$3^{\prime}$ and $5^{\prime}$-GGAGTAGCACCAGAAGAATG-3; amphiregulin: 5'-GTCTCCACTCGCTCTTCC-3' and $5^{\prime}$-GGGCTCTCATTGGTCCTTC-3'; mBD-14: 5'-GTATTCCTCATCTTGTTCTTGG-3' and 5'-AAGGCAGTTAAGTACAGCAC-3'; murine SLPI: 5'-ACGGTGCTCCTTGCTCTG-3' and 5'-GTACGGCATTGTGGCTTCTC-3'; 24p3: 5'-AGGACGACAACATCATCTTCTC-3' and $5^{\prime}$-TGGAGTGGCAGACAGACAG-3'; and murine $\beta$-actin: $5^{\prime}$-ACCCACACTGTGCCCATCTA- $3^{\prime}$ and 5'-CACGCTCGGTCAGGATCTTC- 3 '.

Amplification was performed at $58^{\circ} \mathrm{C}$ for 40 cycles in iCycler Thermal Cycler (Bio-Rad) and data analyzed using iCycler iQ Optical System Software. The relative expression in each sample was calculated by a mathematical method based on the real-time PCR efficiencies (41). 
CFU assays. S. aureus (human clinical isolate, UCLA Clinical Microbiology Facility) and E. coli ML-35p were grown in a shaker incubator at $37^{\circ} \mathrm{C}$ until $\log$ phase in $3 \%$ trypticase soy broth (TSB) (BD). The bacteria were washed once and resuspended in $10 \mathrm{mM}$ phosphate buffer ( $\mathrm{pH} 7.2$ ) with $0.03 \% \mathrm{TSB}$ (S. aureus) or HBSS (Invitrogen) with $0.06 \% \mathrm{TSB}$ (E. coli), and $\mathrm{OD}_{620}$ was adjusted to 0.2 . The bacteria were diluted to a concentration of $1 \times 10^{6} \mathrm{CFU} / \mathrm{ml}$ and incubated with a ratio of $2: 1$ by volume with the epidermal extract in $0.01 \%$ acetic acid for 3 hours at $37^{\circ} \mathrm{C}$. The bacterial solutions were plated on TSB agar plates at various dilutions and incubated overnight at $37^{\circ} \mathrm{C}$; colonies were counted the next day. All experiments were performed in triplicate.

Animal experiments. Five- to six-week-old BALB/c mice were anesthetized, and a dorsal area of the skin was shaved. The shaved area was sterilized with ethanol, and sterile wounding was performed by superficial incisions with a scalpel. The wounded area was covered with OpSite (Medisave) to prevent subsequent bacterial colonization. After 3 days, the mice were sacrificed and the skin from the wounded area and a nonwounded control area was processed for mRNA purification. For the ex vivo wound model, the mice were sacrificed. Skin was surgically removed and cut into slices of $1 \times 10 \mathrm{~mm}$, then grown in keratinocyte medium as described for the human skin slices. Animal experiments were approved by the Ethics Committee at Lund University.
Statistics. Two-tailed paired Student's $t$ test was used.

\section{Acknowledgments}

The outstanding technical assistance of Malgorzata Berlikowski, Maria Baumgarten, and Victoria Gabayan is greatly appreciated. We thank Per Alberius for his outstanding help in supplying skin samples. This work was supported by grants from the Alfred Benzon Foundation, the Novo Nordisk Foundation, the Alfred Österlunds Stiftelse, and NIH grant R01 HL 46809 to T. Ganz. O.E. Sørensen has been a recipient of a Bøje Benzon Fellowship from the Alfred Benzon Foundation and a senior research fellowship from the Novo Nordisk Foundation.

Received for publication March 6, 2006, and accepted in revised form April 25, 2006.

Address correspondence to: Ole E. Sørensen, Section of Clinical and Experimental Infection Medicine, Department of Clinical Sciences, Lund University, Biomedical Center, B14, Tornavägen 10, 22184 Lund, Sweden. Phone: 46-46-222-4472; Fax: 46-46-157-756; E-mail: Ole_E.Sorensen@med.lu.se.
1. Ganz, T. 2002. Epithelia: not just physical barriers. Proc. Natl. Acad. Sci. U. S. A. 99:3357-3358.

2. Zasloff, M. 2002. Antimicrobial peptides of multicellular organisms. Nature. 415:389-395.

3. Nizet, V., et al. 2001. Innate antimicrobial peptide protects the skin from invasive bacterial infection. Nature. 414:454-457.

4. Morrison, G., Kilanowski, F., Davidson, D., and Dorin, J. 2002. Characterization of the mouse Beta defensin 1, defb 1, mutant mouse model. Infect. Immun. 70:3053-3060.

5. Moser, C., et al. 2002. beta-Defensin 1 contributes to pulmonary innate immunity in mice. Infect. Immun. 70:3068-3072.

6. Bals, R., Weiner, D.J., Meegalla, R.L., and Wilson, J.M. 1999. Transfer of a cathelicidin peptide antibiotic gene restores bacterial killing in a cystic fibrosis xenograft model. J. Clin. Invest. 103:1113-1117.

7. Salzman, N.H., Ghosh, D., Huttner, K.M., Paterson, Y., and Bevins, C.L. 2003. Protection against enteric salmonellosis in transgenic mice expressing a human intestinal defensin. Nature. 422:522-526.

8. Lee, P.H.A., et al. 2005. Expression of an additional cathelicidin antimicrobial peptide protects against bacterial skin infection. Proc. Natl. Acad. Sci. U. S. A. 102:3750-3755.

9. Ong, P.Y., et al. 2002. Endogenous antimicrobial peptides and skin infections in atopic dermatitis. N. Engl. J. Med. 347:1151-1160.

10. Nomura, I., et al. 2003. Cytokine milieu of atopic dermatitis, as compared to psoriasis, skin prevents induction of innate immune response genes. J. Immunol. 171:3262-3269.

11. Rieg, S., et al. 2005. Deficiency of dermcidinderived antimicrobial peptides in sweat of patients with atopic dermatitis correlates with an impaired innate defense of human skin in vivo. J. Immunol. 174:8003-8010.

12. Liu, L., Roberts, A.A., and Ganz, T. 2003. By IL-1 signaling, monocyte-derived cells dramatically enhance the epidermal antimicrobial response to lipopolysaccharide. J. Immunol. 170:575-580.

13. Sørensen, O.E., et al. 2005. Differential regulation of $\beta$-defensin expression in human skin by microbial stimuli. J. Immunol. 174:4870-4879.

14. Lemaitre, B., Reichhart, J.-M., and Hoffmann, J.A. 1997. Drosophila host defense: differential induction of antimicrobial peptide genes after infection by various classes of microorganisms. Proc. Natl.
Acad. Sci. U. S. A. 94:14614-14619.

15. Fulton, C., Anderson, G.M., Zasloff, M., Bull, R., and Quinn, A.G. 1997. Expression of natural peptide antibiotics in human skin. Lancet. 350:1750-1751.

16. Harder, J., Bartels, J., Christophers, E., and Schröder, J.-M. 1997. A peptide antibiotic from human skin [letter]. Nature. 387:861.

17. Harder, J., Bartels, J., Christophers, E., and Schröder, J.M. 2001. Isolation and characterization of human beta-defensin-3, a novel human inducible peptide antibiotic. J. Biol. Chem. 276:5707-5713.

18. Mallbris, L., et al. 2002. Neutrophil gelatinaseassociated lipocalin is a marker for dysregulated keratinocyte differentiation in human skin. Exp. Dermatol. 11:584-591.

19. Wingens, M., et al. 1998. Induction of SLPI (ALP/ HUSI-I) in epidermal keratinocytes. J. Invest. Dermatol. 111:996-1002.

20. Ashcroft, G.S., et al. 2000. Secretory leukocyte protease inhibitor mediates non-redundant functions necessary for normal wound healing. Nat. Med. 6:1147-1153.

21. Sørensen, O.E., et al. 2003. Wound healing and expression of antimicrobial peptides/polypeptides in keratinocytes, a consequence of common growth factors. J. Immunol. 170:5583-5589.

22. Blobel, C.P. 2000. Remarkable roles of proteolysis on and beyond the cell surface. Curr. Opin. Cell Biol. 12:606-612.

23. Mitamura, T., Higashiyama, S., Taniguchi, N., Klagsbrun, M., and Mekada, E. 1995. Diphtheria toxin binds to the epidermal growth factor (EGF)like domain of human heparin-binding EGF-like growth factor/diphtheria toxin receptor and inhibits specifically its mitogenic activity. J. Biol. Chem. 270:1015-1019.

24. Gläser, R., et al. 2005. Antimicrobial psoriasin (S100A7) protects human skin from Escherichia coli infection. Nat. Immunol. 6:57-64.

25. Bevins, C.L. 2005. An important clue: fingerprints point to psoriasin in defense against E-coli. Nat. Immunol. 6:12-13.

26. Tokumaru, S., et al. 2000. Ectodomain shedding of epidermal growth factor receptor ligands is required for keratinocyte migration in cutaneous wound healing. J. Cell Biol. 151:209-220.

27. Xu, K.P., Ding, Y., Ling, J.H., Dong, Z., and Yu, F.S.X. 2004. Wound-induced HB-EGF ectodomain shedding and EGFR activation in corneal epithelial cells. Invest. Ophthalmol. Vis. Sci. 45:813-820.

28. Marikovsky, M., et al. 1993. Appearance of hepa- rin-binding EGF-like growth factor in wound fluid as a response to injury. Proc. Natl. Acad. Sci. U. S. A. 90:3889-3893.

29. Singer, A.J., and Clark, R.A. 1999. Cutaneous wound healing. N. Engl. J. Med. 341:738-746.

30. Zetola, N., Francis, J.S., Nuermberger, E.L., and Bishai, W.R. 2005. Community-acquired meticillin-resistant Staphylococcus aureus: an emerging threat. Lancet Infect. Dis. 5:275-286.

31. Park, J.E., and Barbul, A. 2004. Understanding the role of immune regulation in wound healing. Am. J. Surg. 187:11S-16S.

32. Liu, A.Y., et al. 2002. Human beta-defensin-2 production in keratinocytes is regulated by interleukin-1, bacteria, and the state of differentiation. J. Invest. Dermatol. 118:275-281.

33. Sørensen, O.E., et al. 2001. Human cathelicidin, hCAP-18, is processed to the antimicrobial peptide LL-37 by extracellular cleavage with proteinase 3 . Blood. 97:3951-3959.

34. Tjabringa, G.S., et al. 2003. The antimicrobial peptide LL-37 activates innate immunity at the airway epithelial surface by transactivation of the epidermal growth factor receptor. J. Immunol. 171:6690-6696.

35. Zasloff, M. 1987. Margainins, a class of antimicrobial peptides from Xenopus skin: isolation, characterization of two active forms, and partial cDNA sequence precursor. Proc. Natl. Acad. Sci. U. S. A. 84:5449-5453.

36. Simmaco, M., Mignogna, G., and Barra, D. 1998. Antimicrobial peptides from amphibian skin: what do they tell us? Biopolymers. 47:435-450.

37. Brogden, K.A., Guthmiller, J.M., Salzet, M., and Zasloff, M. 2005. The nervous system and innate immunity: the neuropeptide connection. Nat. Immunol. 6:558-564.

38. Sørensen, O.E., et al. 2003. Processing of seminal plasma hCAP-18 to ALL-38 by gastricsin: a novel mechanism of generating antimicrobial peptides in vagina. J. Biol. Chem. 278:28540-28546.

39. Valore, E.V., et al. 1998. Human $\beta$-defensin-1: an antimicrobial peptide of urogenital tissues. J. Clin. Invest. 101:1633-1642.

40. Kjeldsen, L., Koch, C., Arnljots, K., and Borregaard, N. 1996. Characterization of two ELISAs for NGAL, a newly described lipocalin in human neutrophils. J. Immunol. Methods. 198:155-164.

41. Pfaffl, M.W. 2001. A new mathematical model for relative quantification in real-time RT-PCR. Nucleic Acids Res. 29:e45. 